\title{
It's Moving! A Probabilistic Model for Causal Motion Segmentation in Moving Camera Videos
}

\author{
Pia Bideau, Erik Learned-Miller \\ University of Massachusetts, Amherst
}

\begin{abstract}
The human ability to detect and segment moving objects works in the presence of multiple objects, complex background geometry, motion of the observer, and even camouflage. In addition to all of this, the ability to detect motion is nearly instantaneous. While there has been much recent progress in motion segmentation, it still appears we are far from human capabilities. In this work, we derive from first principles a new likelihood function for assessing the probability of an optical flow vector given the 3D motion direction of an object. This likelihood uses a novel combination of the angle and magnitude of the optical flow to maximize the information about the true motions of objects. Using this new likelihood and several innovations in initialization, we develop a motion segmentation algorithm that beats current state-of-the-art methods by a large margin. We compare to five state-of-the-art methods on two established benchmarks, and a third new data set of camouflaged animals, which we introduce to push motion segmentation to the next level.
\end{abstract}

Keywords: motion segmentation, optical flow, moving camera

"Motion is a powerful cue for image and scene segmentation in the human visual system. This is evidenced by the ease with which we see otherwise perfectly camouflaged creatures as soon as they move." -Philip Torr [1]

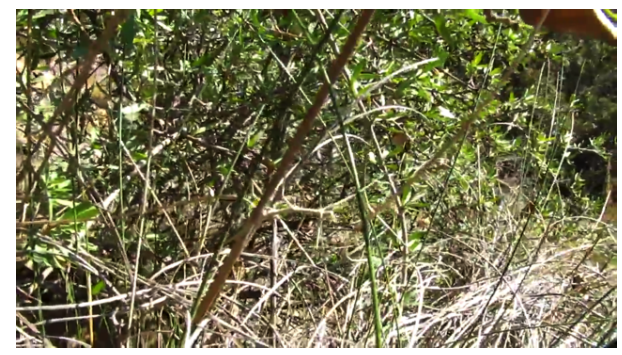

Fig. 1: Where is the camouflaged insect? Before looking at Figure 2, which shows the ground truth localization of this insect, try identifying the insect. While it is virtually impossible to see without motion, it immediately "pops out" to human observers as it moves in the video (see supplementary material).

\section{Introduction}

How can we match the ease and speed with which humans and other animals detect motion? This remarkable capability works in the presence of complex 
background geometry, camouflage, and motion of the observer. Figure 1 is a frame from a video of a "walking stick" insect. Despite the motion of the camera, the rarity of the object, and the high complexity of the background geometry, the insect is immediately visible as soon as it starts moving.

To develop such a motion segmentation system, we re-examined classical methods based upon perspective projection, and developed a new probabilistic model which accurately captures the information about 3D motion in each optical flow vector. In particular, we derive a new conditional flow angle likelihood, $p\left(\mathbf{t}_{\theta} \mid M, \mathbf{t}_{r}\right)$, the probability of observing a particular flow angle $\mathbf{t}_{\theta}$ given the flow magnitude $\mathbf{t}_{r}$ and the 3D motion direction $M$ of an object (for brevity, we will refer to it as the angle likelihood). This angle likelihood is derived from the fundamental perspective projection image formation model, and a model of optical flow as a noisy observation of (2D) scene motion.

This new angle likelihood helps us to address a fundamental difficulty of motion segmentation: the ambiguity of $3 \mathrm{D}$ motion given a set of flow vectors 1 While we cannot eliminate this problem completely, the angle likelihood allows us to weigh the evidence for each image motion properly based on the optical flow. In particular, when the underlying image motion is very small, moderate errors in the optical flow can completely change the apparent motion direction (i.e., the angle of the optical flow vector). When the underlying image motion is large, typical errors in the optical flow will not have a large effect on apparent motion direction. This leads to the critical observation that small optical flow vectors are less informative about motion than large ones. Our derivation of the angle likelihood (Section 3) quantifies this notion and makes it precise in the context of a Bayesian model of motion segmentation.

Fig. 2: Answer: the insect from Figure 1 in shown in red. The insect is trivial to see in the original video, though extremely difficult to identify in a still image. In addition to superior results on standard databases, our method is also one of the few that can detect objects is such complex scenes.

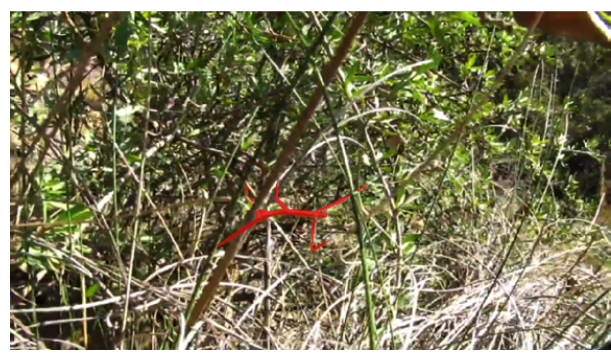

We evaluate our method on three diverse data sets, achieving state-of-the-art performance on all three. The first is the widely used Berkeley Motion Segmentation (BMS-26) database 3, 4, featuring videos of cars, pedestrians, and other common scenes. The second is the Complex Background Dataset [5], designed to test algorithms' abilities to handle scenes with highly variable depth. Third,

$\overline{1}$ Ogale et al. 2] referred to the set of 3D motions compatible with a particular set of optical flow vectors as the motion valley, due to its typical appearance as a long narrow region on the surface of a sphere defining possible motion directions in 3D. 
we introduce a new and even more challenging benchmark for motion segmentation algorithms: the Camouflaged Animal Data Set. The nine (moving camera) videos in this benchmark exhibit camouflaged animals that are difficult to see in a single frame, but can be detected based upon their motion across frames. Our dominance on these three substantially different data sets exhibits the high quality of our method.

\section{Related Work}

A large number of motion segmentation approaches have been proposed, including $3\left[\begin{array}{l}6 \\ 6\end{array} 25\right.$. The prior literature is too large to review here, so we focus on recent methods.

Many methods for motion segmentation work by tracking points or regions through multiple frames to form motion trajectories, and grouping these trajec-

tories into coherent moving objects $3,19,20,22,26$. Elhamifar and Vidal [26] track points through multiple images and show that rigid objects are represented by low-dimensional subspaces in the space of tracks. They use sparse subspace clustering to identify separate objects. Brox and Malik 3 define a pairwise metric on multi-frame trajectories so that they may be clustered to perform motion segmentation. Fragkiadaki et al. 22 detect discontinuities of the embedding density between spatially neighboring trajectories. These discontinuities are used to infer object boundaries and perform segmentation. Papazoglou and Ferrari 19 develop a method that looks both forward and backward in time, using flow angle and flow magnitude discontinuities, appearance modeling, and superpixel mapping across images to connect independently moving objects across frames. Keuper et al. 20] also track points across multiple frames and use minimum cost multicuts to group the trajectories.

Note that these trajectory-based methods are non-causal. To segment earlier frames, they must wait for trajectories which are computed over future frames. Our method, however, is causal, relying only on the flow between two frames and information passed forward from previous frames. Despite this, we outperform trajectory-based methods by a large margin (see Experiments).

Another set of methods analyze optical flow between a pair of frames, grouping pixels into regions whose flow is consistent with various motion models. Torr [1] develops a sophisticated probabilistic model of optical flow, building a mixture model that explains an arbitrary number of rigid components within the scene. Interestingly, he assigns different types of motion models to each object based on model fitting criteria. His approach is fundamentally based on projective geometry rather based directly on perspective projection equations, as in our approach. Horn has identified drawbacks of using projective geometry in such estimation problems and has argued that methods based directly on perspective projection are less prone to overfitting in the presence of noise 27]. Zamalieva et al. 18 present a combination of methods that rely on homographies and fundamental matrix estimation. The two methods have complimentary strengths, and the authors attempt to select among the best dynamically. An advantage 
of our method is that we do not depend upon the geometry of the scene to be well-approximated by a group of homographies, which enables us to address videos with very complex background geometries. Narayana et al. 17 remark that for translational only motions, the angle field of the optical flow will consist of one of a set of canonical angle fields, one for each possible motion direction, regardless of the focal length. They use these canoncial angle fields as a basis with which to segment a motion image. However, they do not handle camera rotation, which is a significant limitation.

Another set of methods using occlusion events in video to reason about depth ordering and independent object motion [2,21]. Ogale et al. [2] use occlusion cues to further disambiguate non-separable solutions to the motion segmentation problem. Taylor et al. 21] introduce a causal framework for integrating occlusion cues by exploiting temporary consistency priors to partition videos into depth layers.

Estimation of a camera's translation and rotation from the observed optical flow is closely related to motion segmentation 28 34. For lack of space, we discuss these works in Supp. Mat.

\section{Methods}

The motion field of a scene is created by the movement of the camera relative to a stationary background and the additional motion of independently moving objects. We use the optical flow, or estimated motion field, to segment each video image into background and other independently moving objects.

When the camera is only translating (and not rotating) relative to the background, there are strong constraints on the background's optical flow-the $d i$ rection or angle $\boldsymbol{t}_{\boldsymbol{\theta}}$ of the motion at each pixel is determined by the camera translation $(U, V, W)$, the image location of the pixel $(x, y)$, and the camera's focal length $f$, and has no dependence on scene depth [35.

$$
\boldsymbol{t}_{\boldsymbol{\theta}}=\arctan (W \cdot y-V \cdot f, W \cdot x-U \cdot f)
$$

Simultaneous camera rotation and translation, however, couple the scene depth and the optical flow, making it much harder to assign pixels to the right motion model.

To address this, we wish to subtract off the estimated rotational component $\hat{O}_{R}$ of optical flow from the original flow $O$ to produce a translation component estimate $\hat{O}_{T}$. The subsequent assignment of flow vectors to motion models is thus greatly simplified. However estimating camera rotation in the presence of multiple motions is challenging. We organize the Methods section as follows.

In Section 3.1. we describe how all frames after the first frame are segmented, using the segmentation from the previous frame and our novel angle likelihood. After reviewing Bruss and Horn's motion estimation technique [36] in Section 3.2. Section 3.3 describes how our method is initialized in the first frame, including a novel process for estimating camera motion in the presence of multiple motions. 


\subsection{A probabilistic model for motion segmentation}

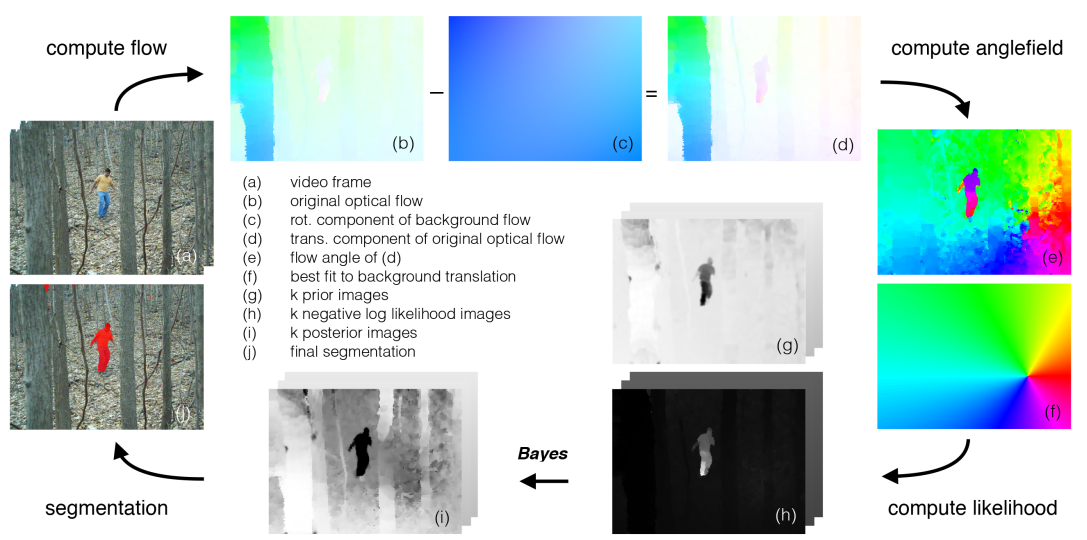

Fig. 3: Our segmentation procedure. Given the optical flow (b) the camera motion is estimated. Then, the rotational flow $\hat{O}_{R}(\mathrm{c})$ is subtracted from the optical flow $O$ to produce a translational flow $\hat{O}_{T}$. The angle field of $\hat{O}_{T}$ is shown in (e). The best fitting translation parameters to the background of $\hat{O}_{T}$ yield an angle field (f), which clearly shows the forward motion of the camera (rainbow focus of expansion pattern) not visible in the original angle field. The motion component priors $(\mathrm{g})$ and negative $\log$ likelihoods $(\mathrm{h})$ yield the posteriors (i) and the final segmentation $(\mathrm{j})$.

Given a (soft) motion segmentation of frame $T-1$ into $k$ moving objects and the optical flow $O$ from frames $T$ and $T+1$, segmenting frame $T$ requires several ingredients: a) the prior probabilities $p\left(M_{j}\right)$ for each pixel that it is assigned to each particular motion model $\left.M_{j}, \mathbf{b}\right)$ an estimate of the current 3D motion directions $M_{j}, 1 \leq j \leq k$, for each of the $k$ objects from the previous frame, c) for each pixel position, an angle likelihood $p\left(\mathbf{t}_{\theta} \mid M_{j}, \mathbf{t}_{r}\right)$ that gives the likelihood of each flow angle $\mathbf{t}_{\theta}$ under each motion model $M_{j}$ conditioned on the flow magnitude $\mathbf{t}_{r}$, and $\mathbf{d}$ ) the prior probability $p\left(M_{k+1}\right)$ and angle likelihoods $p\left(\boldsymbol{t}_{\theta} \mid M_{k+1}, \boldsymbol{t}_{r}\right)$ of a new motion $M_{k+1}$. Given these priors and likelihoods, we simply use Bayes' rule to obtain posterior probabilities of each motion at each pixel location. We have

$$
\begin{aligned}
p\left(M_{j} \mid \boldsymbol{t}_{\theta}, \boldsymbol{t}_{r}\right) & \propto p\left(\boldsymbol{t}_{\theta} \mid M_{j}, \boldsymbol{t}_{r}\right) \cdot p\left(M_{j} \mid \boldsymbol{t}_{r}\right) \\
& =p\left(\boldsymbol{t}_{\theta} \mid M_{j}, \boldsymbol{t}_{r}\right) \cdot p\left(M_{j}\right) .
\end{aligned}
$$

The second expression follows since the prior $M_{j}$ does not depend on $\boldsymbol{t}_{r}$. We directly use this posterior for segmentation. We now describe how the above quantities are computed.

Propagating the posterior for a new prior. We start from the optical flow of Sun et al. 37. (Figure 3b). We then create a prior at each pixel for each 
motion model in the new frame (Figure $3 \mathrm{~g}$ ) by propagating the posterior from the previous frame (Figure 3 ) in three steps.

- Use the previous frame's flow to map posteriors from frame $T-1$ (Figure 3 ) to new positions in frame $T$.

- Smooth the mapped posterior in the new frame by convolving with a spatial Gaussian, as done in 17,38. This implements the idea that object locations in future frames are likely to be close to their locations in previous frames.

- Renormalize the smoothed posterior from the previous frame to form a proper probability distribution at each pixel location, which acts as the prior on the $k$ motion components for the new frame (Figure $3 \mathrm{~g}$ ). Finally, we set aside a probability of $1 /(k+1)$ for the prior of a new motion component, while rescaling the priors for the pre-existing motions to sum to $k /(k+1)$.

Estimating and removing rotational flow. We use the prior for the background component to weight pixels for estimating the current frame's flow due to the camera. ${ }^{2}$ We estimate the camera translation parameters $(U, V, W)$ and rotation parameters $(A, B, C)$ using a modified version of the Bruss and Horn algorithm 36 (Section 3.2. As described above, we then render the flow angle independent of the unknown scene depth by subtracting the estimated rotational component $\hat{O}_{R}$ (Figure 3 ) from the original flow $O$ (Figure 3 ) to produce an estimate of the translational flow $\hat{O}_{T}$ (Fig. $\left.3 \mathrm{~d}\right)$ :

$$
\hat{O}_{T}=O-\hat{O}_{R}(\hat{A}, \hat{B}, \hat{C}) \text {. }
$$

We denote by $\boldsymbol{t}$ the estimated translation at a particular pixel. Next, for each addtitional motion component, we estimate 3D translation direction parameters using the segment priors to select pixels, weighted according to the prior. Let the collection of translation parameters for motion component $j$ be denoted $M_{j}$.

The flow angle likelihood. Once we have obtained $\hat{O}_{T}$ by removing the rotational flow, we use each flow vector $\boldsymbol{t}$ to decide which motion component it belongs to. Most of the information about the 3D motion direction is contained in the flow angle, not the flow magnitude. This is because for a given translational 3D motion direction (relative to the camera), the flow angle is completely determined by that motion and the location in the image, whereas the flow magnitude is a function of the object's depth, which is unknown. However, as discussed above, the amount of information in the flow angle depends upon the flow magnitude-flow vectors with greater magnitude are much more reliable indicators of true motion direction. This is why it is critical to formulate the angle likelihood conditioned on the flow magnitude.

Other authors have used flow angles in motion segmentation. For example, Papazoglou and Ferrari 19] use both a gradient of the optical flow and a separate function of the flow angle to define motion boundaries. Narayana et al. [17] use only the optical flow angle to evaluate motions. But our derivation gives a principled, novel, and highly effective method of using the flow angle and magnitude

${ }^{2}$ Note that we do not explicitly estimate the camera motion, since we know neither the speed nor focal length of the camera, only the direction of motion. 
together to mine accurate information from the optical flow. In particular, we show that while (under certain mild assumptions) the translational magnitudes alone have no information about which motion is most likely, the magnitudes play an important role in specifying the informativeness of the flow angles. In our experiments section, we demonstrate that failing to condition on flow magnitudes in this way results in greatly reduced performance over our derived model.

We now derive the most important element of our method, a high quality conditional flow angle likelihood $p\left(\boldsymbol{t}_{\theta} \mid M_{j}, \boldsymbol{t}_{r}\right)$, the probability of observing a flow direction $\boldsymbol{t}_{\theta}$ given that a pixel was part of an object undergoing motion $M_{j}$, and that the flow magnitude was $\boldsymbol{t}_{r}$. We make the following modeling assumptions:

1. We assume the observed translational flow $\boldsymbol{t}=\left(\boldsymbol{t}_{r}, \boldsymbol{t}_{\theta}\right)$ at a pixel is a noisy observation of the unobserved translational motion field $\boldsymbol{t}^{*}=\left(\boldsymbol{t}_{r}^{*}, \boldsymbol{t}_{\theta}^{*}\right)$ :

$$
\boldsymbol{t}=\boldsymbol{t}^{*}+\eta
$$

where $\eta$ is independent 2D Gaussian noise with 0-mean and circular but unknown covariance $s \cdot I$.

2. We assume the translational motion field magnitude $\boldsymbol{t}_{r}^{*}$ is statistically independent of the translation motion field angle $\boldsymbol{t}_{\theta}^{*}$. It follows that $\boldsymbol{t}_{r}=\boldsymbol{t}_{r}^{*}+\eta$ is also independent of $\boldsymbol{t}_{\theta}^{*}$, and hence $p\left(\boldsymbol{t}_{r} \mid \boldsymbol{t}_{\theta}^{*}\right)=p\left(\boldsymbol{t}_{r}\right)$.

3. A motion model $M_{j}$ gives the direction of $3 \mathrm{D}$ motion, but not its magnitude. For a given position in the image, each $3 \mathrm{D}$ motion direction yields a (2D) motion field direction $\boldsymbol{t}_{\theta}^{*}$ in the image. We assume that $\boldsymbol{t}_{\theta}^{*}$ contains all of the information about the motion model useful for predicting the optical flow, or $p\left(\boldsymbol{t} \mid M_{j}\right)=p\left(\boldsymbol{t} \mid \boldsymbol{t}_{\theta}^{*}\right)$.

With these assumptions, we have

$$
\begin{aligned}
p\left(\boldsymbol{t} \mid M_{j}\right) & \stackrel{(3)}{=} p\left(\boldsymbol{t} \mid \boldsymbol{t}_{\theta}^{*}\right) \\
& \stackrel{(1)}{=} p\left(\boldsymbol{t}_{r}, \boldsymbol{t}_{\theta} \mid \boldsymbol{t}_{\theta}^{*}\right) \\
& =p\left(\boldsymbol{t}_{\theta} \mid \boldsymbol{t}_{r}, \boldsymbol{t}_{\theta}^{*}\right) \cdot p\left(\boldsymbol{t}_{r} \mid \boldsymbol{t}_{\theta}^{*}\right) \\
& \stackrel{(2)}{=} p\left(\boldsymbol{t}_{\theta} \mid \boldsymbol{t}_{r}, \boldsymbol{t}_{\theta}^{*}\right) \cdot p\left(\boldsymbol{t}_{r}\right) \\
& \propto p\left(\boldsymbol{t}_{\theta} \mid \boldsymbol{t}_{r}, \boldsymbol{t}_{\theta}^{*}\right) \\
& \stackrel{(3)}{=} p\left(\boldsymbol{t}_{\theta} \mid M_{j}, \boldsymbol{t}_{r}\right),
\end{aligned}
$$

where the numbers over each equality give the assumption that is invoked. Equation (10) follows since $p\left(\boldsymbol{t}_{r}\right)$ is constant across all motion models.

We model $p\left(\boldsymbol{t}_{\theta} \mid \boldsymbol{t}_{r}, \boldsymbol{t}_{\theta}^{*}\right)$ using a von Mises distribution with parameters $\mu$, the preferred direction, and concentration parameter $\kappa$. We set $\mu=\boldsymbol{t}_{\theta}^{*}$, since the most likely observed angle $\boldsymbol{t}_{\theta}$ is the ground truth angle $\boldsymbol{t}_{\theta}^{*}$. To set $\kappa$, we observe that when the ground truth flow magnitude $\boldsymbol{t}_{r}^{*}$ is small, the distribution of observed angles $\boldsymbol{t}_{\theta}$ will be near uniform (see Figure $4, \boldsymbol{t}^{*}=(0,0)$ ), whereas when $\boldsymbol{t}_{r}^{*}$ is large, the observed angle $\boldsymbol{t}_{\theta}$ is likely to be close to the ground truth 

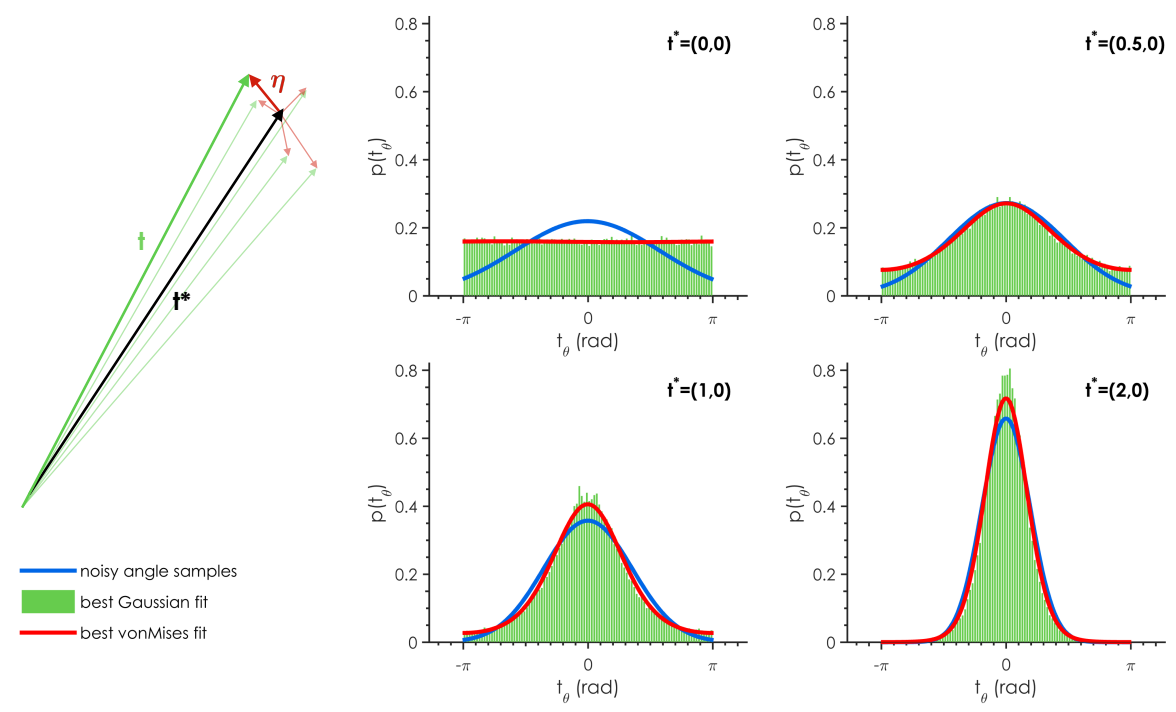

Fig. 4: The von Mises distribution. When a motion field vector $\boldsymbol{t}^{*}$ is perturbed by added Gaussian noise $\eta$ (figure top left), the resulting distribution over optical flow angles $\boldsymbol{t}_{\theta}$ is well-modeled by a von Mises distribution. The figure shows how small motion field vectors result in a broad distribution of angles after noise is added, while larger magnitude motion field vectors result in a narrower distribution of angles. The red curve shows the best von Mises fit to these sample distributions and the blue curve shows the lower quality of the best Gaussian fit.

flow angle $\boldsymbol{t}_{\theta}^{*}$ (Figure $4, \boldsymbol{t}^{*}=(2,0)$ ). We can achieve this basic relationship by setting $\kappa=a\left(\boldsymbol{t}_{r}^{*}\right)^{b}$, where $a$ and $b$ are parameters that give added flexibility to the model. Since we don't have direct access to $\boldsymbol{t}_{r}^{*}$, we use $\boldsymbol{t}_{r}$ as a surrogate, yielding

$$
p\left(\boldsymbol{t} \mid M_{j}\right) \propto \operatorname{vonMises}\left(\boldsymbol{t}_{\theta} ; \mu=\boldsymbol{t}_{\theta}^{*}, \kappa=a \boldsymbol{t}_{r}{ }^{b}\right) .
$$

Note that this likelihood treats zero-length translation vectors as uninformativeit assigns them the same likelihood under all motions. This makes sense, since the direction of a zero-length optical flow vector is essentially random. Similarly, the longer the optical flow vector, the more reliable and informative it becomes.

Likelihood of a new motion. Lastly, with no prior information about new motions, we set $p\left(\boldsymbol{t}_{\theta} \mid M_{k+1}\right)=\frac{1}{2 \pi}$, a uniform distribution.

Once we have priors and likelihoods, we compute the posteriors (Equation 2 and label each pixel as

$$
L=\underset{j}{\arg \max } p\left(M_{j} \mid \boldsymbol{t}_{\theta}, \boldsymbol{t}_{r}\right) .
$$

\subsection{Bruss and Horn's motion estimation.}

To estimate the direction of motion (but not the speed) of the camera relative to the background, we use the method of Bruss and Horn [36] and apply it to 
pixels selected by the background prior. The optical flow vector $\boldsymbol{v}_{i}$ at pixel $i$ can be decomposed as $\boldsymbol{v}_{i}=\boldsymbol{p}_{i}+\boldsymbol{e}_{i}$, where $\boldsymbol{p}_{i}$ is the component of $\boldsymbol{v}_{i}$ in the direction predicted by the motion model and $\boldsymbol{e}_{i}$ is the component orthogonal to $\boldsymbol{p}_{i}$. The authors find the motion $M$ that minimizes the sum of these "error" components $\boldsymbol{e}_{i}$. The optimization for translation-only is

$$
\underset{U, V, W}{\arg \min } \sum_{i}\left\|\boldsymbol{e}_{i}\left(\boldsymbol{v}_{i}, U, V, W\right)\right\|
$$

where $U, V$, and $W$ are the three translational motion components. Bruss and Horn give a closed form solution to this problem for the translation-only case.

Recovering camera rotation. Bruss and Horn also outline how to solve for rotation, but give limited details. We implement our own estimation of rotations $(A, B, C)$ and translation as a nested optimization:

$$
\hat{M}=\underset{A, B, C, U, V, W}{\arg \min }\left[\min _{U, V, W} \sum_{i}\left\|\boldsymbol{e}_{i}\left(\boldsymbol{v}_{i}, A, B, C, U, V, W\right)\right\|\right] .
$$

Given the portion $\hat{O}_{R}$ of the observed flow $O$ due to rotation, one can subtract off the rotation since it does not depend on scene geometry: $\hat{O}_{T}=O-\hat{O}_{R}$.

Subtracting the rotation $(A, B, C)$ from the observed flow reduces the optimization to the translation only case. We solve the optimization over the rotation parameters $A, B, C$ by using Matlab's standard gradient descent optimization, while calling the Bruss and Horn closed form solution for the translation variables given the rotational variables as part of the internal function evaluation. Local minima are a concern, but since we are estimating camera motion between two video frames, the rotation is almost always small and close to the optimization's starting point.

\subsection{Initialization: Segmenting the first frame}

The goals of the initialization are a) estimating background translation and rotation parameters, b) finding pixels whose flow is consistent with this motion model, and c) assigning inconsistent groups of contiguous pixels to additional motion models. Bruss and Horn's method was not developed to handle scenes with multiple different motions, and so large or fast-moving foreground objects can result in poor motion estimates (Figure 7).

Constrained RANSAC. To address this problem we use a modified version of RANSAC [39] to robustly estimate background motion (Figure 5). We use 10 random SLIC superpixels 40$]^{3}$ to estimate camera motion (Section 3.2. We modify the standard RANSAC procedure to force the algorithm to choose three of the 10 patches from the image corners, because image corners are prone to errors due to a misestimated camera rotation. Since the Bruss and Horn error

${ }^{3}$ We use the http://www.vlfeat.org/api/slic.html code with regionSize $=20$ and regularizer $=0.5$. 
function (Equation 15) does not penalize motions in a direction opposite of the predicted motion, we modify it to penalize these motions appropriately (details in Supp. Mat.). 5000 RANSAC trials are run, and the camera motion $\hat{M}$ resulting in the fewest outlier pixels according to the modified Bruss-Horn (MBH) error is retained, using a threshold of 0.1 .

Input: video with $n$ frames

Output: binary motion segmentation

for $T \leftarrow 1$ to $n-1$ do

compute opticalflow from frame $T$ to frame $T+1$;

if first frame then

foreach RANSAC iteration do

find best motion model for 10 random patched ( 3 in corners);

retain best motion model for the static backgound $M_{b}$; end

$p\left(M_{j}\right), p\left(M_{b}\right) \leftarrow$ segment $\mathrm{MBH}$ error image into $k$ components

using Otsu's method;

else

$p\left(M_{j}\right), p\left(M_{b}\right) \leftarrow$ propagate posterior $p\left(M_{j} \mid \boldsymbol{t}\right), p\left(M_{b} \mid \boldsymbol{t}\right)$;

compute motion model $M_{b}$ of static background;

end

foreach pixel in $\hat{O}_{T}$ do

$\mid p\left(\boldsymbol{t}_{\theta} \mid M_{b}, \boldsymbol{t}_{r}\right) \leftarrow \operatorname{vonMises}\left(\boldsymbol{t}_{\theta} ; \mu\left(\boldsymbol{t}_{\theta}^{*}\right), \kappa\left(\boldsymbol{t}_{r}\right)\right) ;$

end

for $j \leftarrow 1$ to $k-1$ do

compute motion model $M_{j}$ of moving object $j$;

foreach pixel in $\hat{O}_{T}$ do

$\underset{\text { end }}{\mid} p\left(\boldsymbol{t}_{\theta} \mid M_{j}, \boldsymbol{t}_{r}\right) \leftarrow \operatorname{vonMises}\left(\boldsymbol{t}_{\theta} ; \mu\left(\boldsymbol{t}_{\theta}^{*}\right), \kappa\left(\boldsymbol{t}_{r}\right)\right) ;$

end

foreach pixel in $\hat{O}_{T}$ do

$p\left(M_{k+1}\right) \leftarrow \frac{1}{k+1}$

$p\left(\boldsymbol{t}_{\theta} \mid M_{k+1}, \boldsymbol{t}_{r}\right) \leftarrow \frac{1}{2 \pi}$

normalize $p\left(M_{b}\right)$ and $p\left(M_{j}\right)$ such that they sum up to $1-p\left(M_{k+1}\right)$; $p(M \mid \boldsymbol{t}) \leftarrow p\left(\boldsymbol{t}_{\theta} \mid M, \boldsymbol{t}_{r}\right) \cdot p(M) ;$

end

given the posteriors $p\left(M_{b} \mid \boldsymbol{t}\right), p\left(M_{j} \mid \boldsymbol{t}\right)$ and $p\left(M_{k+1} \mid \boldsymbol{t}\right)$ assign every pixel one of two labels: static background or moving objects;

end

Algorithm 1: A causal motion segmentation algorithm

Otsu's Method. While using the RANSAC threshold on the MBH image produces a good set of pixels with which to estimate the background motion, the method often excludes some pixels that should be included in the background motion component. We use Otsu's method [41] to separate the MBH image into a region of low error (background) and high error: 
- Use Otsu's threshold to divide the errors, minimizing the intraclass variance. Use this threshold to do a binary segmentation of the image.

- Find the connected component $C$ with highest average error. Remove these pixels $(I \leftarrow I \backslash C)$, and assign them to an additional motion model.

These steps are repeated until Otsu's effectiveness parameter is below 0.6.
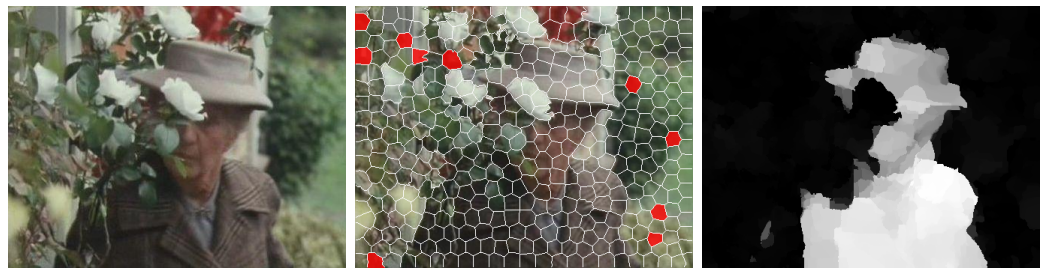

Fig. 5: RANSAC procedure. The result of our RANSAC procedure to find background image patches. Notice that none of the patches are on the person moving in the foreground. Also notice that we force the algorithm to pick patches in three of the four image corners (a "corner" is $4 \%$ of the image). The right figure shows the negative log likelihood of background.

\section{Experiments}

Several motion segmentation benchmarks exist, but often a clear definition of what people intend to segment in ground truth is missing. The resulting inconsistent segmentations complicate the comparison of methods. We define motion segmentation as follows.

(I) Every pixel is given one of two labels: static background or moving objects.

(II) If only part of an object is moving (like a moving person with a stationary foot), the entire object should be segmented.

(III) All freely moving objects (not just one) should be segmented, but nothing else. We do not considered tethered objects such as trees to be freely moving.

(IV) Stationary objects are not segmented, even when they moved before or will move in the future. We consider segmentation of previously moving objects to be tracking. Our focus is on segmentation by motion analysis.

Experiments were run on two previous datasets and our new camouflaged animals videos. The first was the Berkeley Motion Segmentation (BMS-26) database [3,4] (Figure 8, rows 5,6). Some BMS videos have an inconsistent definition of ground truth from both our definition and from the other videos in the benchmark. An example is Marple10 whose ground truth segments a wall in the foreground as a moving object (see Figure 6). While it is interesting to use camera motion to segment static objects (as in [42]), we are addressing the segmentation of objects that are moving differently than the background, and so we 
excluded ten such videos from our experiments (see Supp. Mat.). The second database used is the Complex Background Data Set [17], which includes significant depth variation in the background and also signficant amounts of camera rotation (Figure 8, rows 3,4). We also introduce the Camouflaged Animals Data Set (Figure 8, rows 1,2) which will be released at camera-ready time. These videos were ground-truthed every 5th frame. See Supp. Mat. for more.

Fig. 6: Bad ground truth. Some BMS-26 videos contain significant ground truth errors, such as this segmentation of the foreground wall, which is clearly not a moving object.
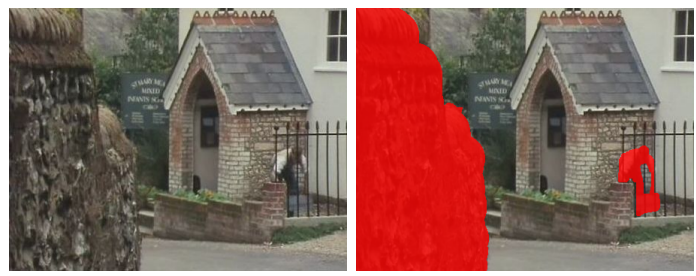

Setting von Mises parameters. There are two parameters $a$ and $b$ that affect the von Mises concentration $\kappa=a r^{b}$. To set these parameters for each video, we train on the remaining videos in a leave-one-out paradigm, maximizing over the values $0.5,1.0,2.0,4.0$ for multiplier parameter $a$ and the values $0,0.5,1,2$ for the exponent parameter $b$. Cross validation resulted in the selection of the parameter pair $(a=4.0, b=1.0)$ for most videos, and we adopted these as our final values.

\begin{tabular}{|c|c|c|c|c|c|c|c|}
\hline & & $\begin{array}{c}\text { Keuper } \\
20\end{array}$ & $\begin{array}{c}\text { Papaz. } \\
{[19]}\end{array}$ & $\begin{array}{r}\text { Frag. } \\
{[22}\end{array}$ & $\underset{18}{\text { Zama. }}$ & $\begin{array}{c}\text { Naray. } \\
17\end{array}$ & ours \\
\hline \multirow[t]{2}{*}{ Camouflage } & MCC & 0.4305 & 0.3517 & 0.1633 & 0.3354 & - & 0.5344 \\
\hline & $\mathrm{F}$ & 0.4379 & 0.3297 & 0.1602 & 0.3007 & - & 0.5276 \\
\hline \multirow[t]{2}{*}{ BMS-26 } & MCC & 0.6851 & 0.6112 & 0.7187 & 0.6399 & - & 0.7576 \\
\hline & $\mathrm{F}$ & 0.7306 & 0.6412 & 0.7276 & 0.6595 & 0.6246 & 0.7823 \\
\hline \multirow[t]{2}{*}{ Complex } & MCC & 0.4752 & 0.6359 & 0.3257 & 0.3661 & - & 0.7491 \\
\hline & $\mathrm{F}$ & 0.4559 & 0.6220 & 0.3300 & 0.3297 & 0.3751 & 0.7408 \\
\hline \multirow{2}{*}{ Total avg. } & MCC & 0.5737 & 0.5375 & 0.4866 & 0.5029 & - & 0.6918 \\
\hline & $\mathrm{F}$ & 0.5970 & 0.5446 & 0.4911 & 0.4969 & - & 0.6990 \\
\hline
\end{tabular}

Table 1: Comparison to state-of-the-art. Matthew's correlation coefficient and Fmeasure for each method and data set. The "Total avg." numbers average across all valid videos.

Results. In Tab. 1 we compare our model to five different state-of-the-art methods $17,20,22]$. We compared against methods for which either code was available or that had results on either of the two public databases that we used. However, we excluded some methods (such as [21]), as their published results were less accurate than [20], to whom we compared. 
Some authors have scored algorithms using the number of correctly labeled pixels. However, when the moving object in the foreground is small, a method can achieve a very high score simply by marking the entire video as background. The F-measure is also not symmetric with respect to foreground and background, and is not well-defined when a frame contains no foreground pixels. Matthew's Correlation Co-efficient (MCC) handles both of these issues, and is recommended for scoring such binary classification problems when there is a large imbalance between the number of pixels in each category [43]. However, in order to enable comparison with [17], and to allow easier comparison to other methods, we also included F-measures. Table 1 shows the highest average accuracy per data set in green and the second best in blue, for both the F-measure and MCC. We were not able to obtain code for Narayana et al. [17, but reproduced F-measures directly from their paper. The method of 22 failed on several videos (only in the BMS data set), possibly due to the length of these videos. In these cases, we assigned scores for those videos by assigning all pixels to background.

Our method outperforms all other methods by a large margin, on all three data sets, using both measures of comparison.

\section{$5 \quad$ Analysis and Conclusions}

Conditioning our angle likelihood on the flow magnitude is an important factor in our method. Table 2 shows the detrimental effect of using a constant von Mises concentration $\kappa$ instead of one that depends upon flow magnitude. In this experiment, we set the parameter $b$ which governs the dependence of $\kappa$ on $\boldsymbol{t}_{r}$ to 0 , and set the value of $\kappa$ to maximize performance. Even with the optimum constant $\kappa$, the drop in performance was $7 \%, 5 \%$, and a whopping $22 \%$ across the three data sets.

\begin{tabular}{lccc}
\hline & final & constant $\kappa$ & no RANSAC \\
\hline BMS-26 & 0.7576 & 0.6843 & 0.6450 \\
complex & 0.7491 & 0.7000 & 0.5757 \\
camouflage & 0.5344 & 0.3128 & 0.5176 \\
\hline
\end{tabular}

Table 2: Effect of RANSAC and variable $\kappa$.

We also show the consistent gains stemming from our constrained RANSAC initialization procedure. In this experiment, we segmented the first frame of video without rejecting any pixels as outliers. In some videos, this had little effect, but sometimes the effect was large, as shown in Figure 7.

The method by Keuper et al. 20 performs fairly well, but often makes errors in segmenting rigid parts of the foreground near the observer. This can be seen in the third and fourth rows of Figure 8, which shows sample results from the Complex Background Data Set. In particular, note that Keuper et al.'s method 


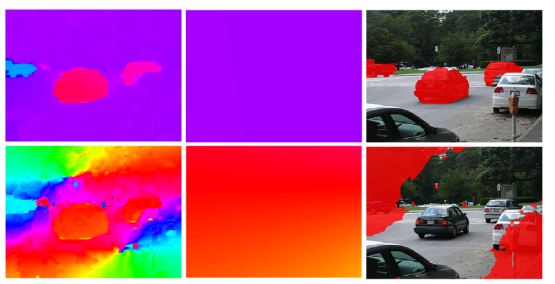

Fig. 7: RANSAC vs no RANSAC. Top row: robust initialisation with RANSAC. Bottom row: using Bruss and Horn's method directly on the entire image. Left to right: flow angles of translational flow, flow angles of estimated background translation and segmentation. Note that without RANSAC the estimated background translation is the best fit for the car instead of background

segments the tree in the near foreground in the third row and the wall in the near foreground in the fourth row. The method of Fragkiadaki et al., also based on trajectories, has similar behavior. These methods in general seem to have difficulty with high variability in depth.

Another reason for our excellent performance may be that we are not making any compromises in modeling motion. We are directly using the perspective projection equations to analyze motion, as has been advocated by Horn 27], rather than approximations based on projective geometry, as done by Zamalieva et al. Code is available: http://vis-www.cs.umass.edu/motionSegmentation/

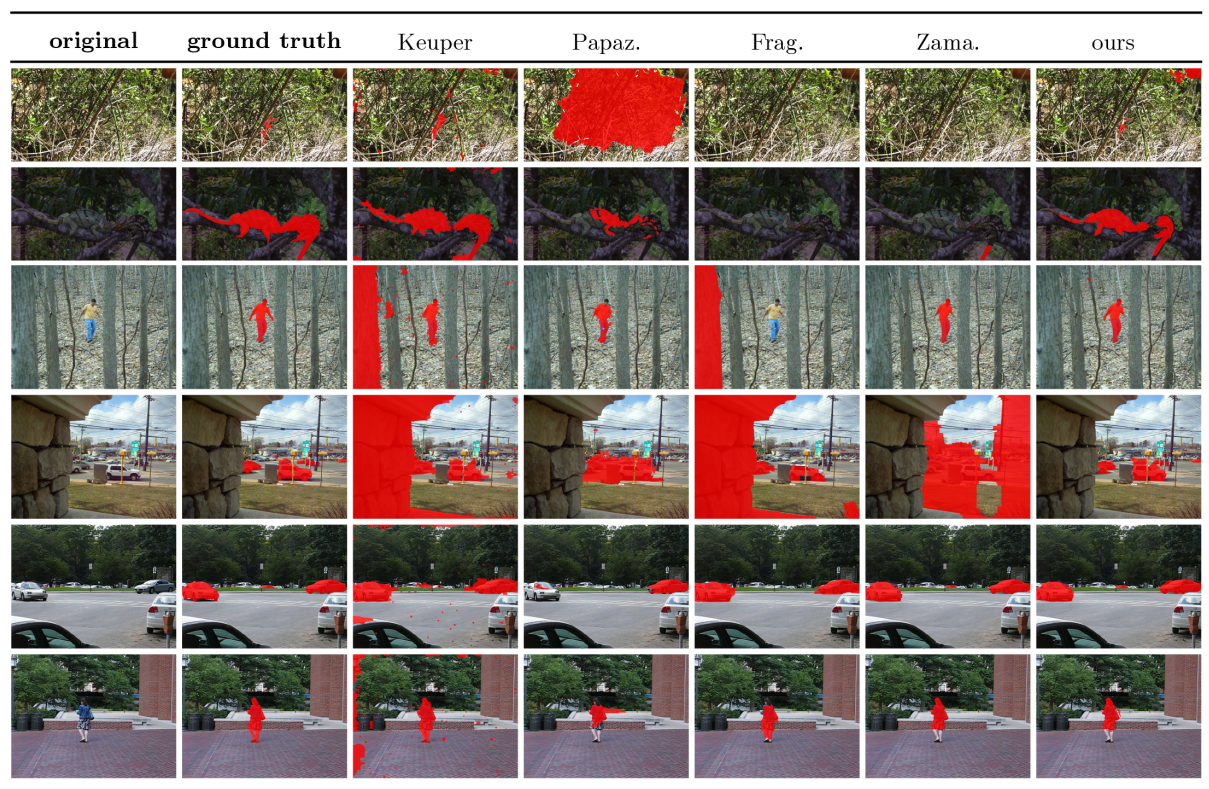

Fig. 8: Sample results Left to right: original image, ground truth, 20, 19, 22,18 and our binary segmentations. Rows 1-2: sample results on the Animal Camouflage Data Set (chameleon and stickinsect). Rows 3-4: sample results on Complex Background (traffic and forest). Rows 5-6: sample results on BMS-26 (cars5 and people1). 


\section{References}

1. Torr, P.H.: Geometric motion segmentation and model selection. Philosophical Transactions of the Royal Society of London A: Mathematical, Physical and Engineering Sciences 356(1740) (1998) 1321-1340

2. Ogale, A.S., Fermüller, C., Aloimonos, Y.: Motion segmentation using occlusions. Pattern Analysis and Machine Intelligence, IEEE Transactions on 27(6) (2005) 988-992

3. Brox, T., Malik, J.: Object segmentation by long term analysis of point trajectories. In: ECCV. (2010)

4. Tron, R., Vidal, R.: A benchmark for the comparison of 3-d motion segmentation algorithms. In: CVPR. (2007)

5. Narayana, M., Hanson, A., Learned-Miller, E.: Coherent motion segmentation in moving camera videos using optical flow orientations. In: ICCV. (2013)

6. Grundmann, M., Kwatra, V., Han, M., Essa, I.: Efficient hierarchical graph-based video segmentation. In: Computer Vision and Pattern Recognition (CVPR), 2010 IEEE Conference on, IEEE (2010) 2141-2148

7. Lezama, J., Alahari, K., Sivic, J., Laptev, I.: Track to the future: Spatio-temporal video segmentation with long-range motion cues. In: Proceedings of the IEEE Conference on Computer Vision and Pattern Recognition. (2011)

8. Kumar, M.P., Torr, P.H., Zisserman, A.: Learning layered motion segmentations of video. International Journal of Computer Vision 76(3) (2008) 301-319

9. Irani, M., Rousso, B., Peleg, S.: Computing occluding and transparent motions. International Journal of Computer Vision 12 (1994) 5-16

10. Ren, Y., Chua, C.S., Ho, Y.K.: Statistical background modeling for non-stationary camera. Pattern Recognition Letters 24 (2003) 183 - 196

11. Sheikh, Y., Javed, O., Kanade, T.: Background subtraction for freely moving cameras. In: IEEE 12th International Conference on Computer Vision, ICCV 2009, Kyoto, Japan, September 27 - October 4, 2009, IEEE (2009) 1219-1225

12. Elqursh, A., Elgammal, A.M.: Online moving camera background subtraction. In: ECCV. (2012)

13. Ochs, P., Brox, T.: Higher order motion models and spectral clustering. In: CVPR. (2012)

14. Kwak, S., Lim, T., Nam, W., Han, B., Han, J.H.: Generalized background subtraction based on hybrid inference by belief propagation and Bayesian filtering. In: ICCV. (2011)

15. Rahmati, H., Dragon, R., Aamo, O.M., Van Gool, L., Adde, L.: Motion segmentation with weak labeling priors. In: Pattern Recognition. Springer (2014) 159-171

16. Jain, S.D., Grauman, K.: Supervoxel-consistent foreground propagation in video. In: Computer Vision-ECCV 2014. Springer (2014) 656-671

17. Narayana, M., Hanson, A., Learned-Miller, E.: Coherent motion segmentation in moving camera videos using optical flow orientations. In: Computer Vision (ICCV), 2013 IEEE International Conference on, IEEE (2013) 1577-1584

18. Zamalieva, D., Yilmaz, A., Davis, J.W.: A multi-transformational model for background subtraction with moving cameras. In: Computer Vision-ECCV 2014. Springer (2014) 803-817

19. Papazoglou, A., Ferrari, V.: Fast object segmentation in unconstrained video. In: Computer Vision (ICCV), 2013 IEEE International Conference on, IEEE (2013) $1777-1784$ 
20. Keuper, M., Andres, B., Brox, T.: Motion trajectory segmentation via minimum cost multicuts. In: Proceedings of the IEEE International Conference on Computer Vision. (2015) 3271-3279

21. Taylor, B., Karasev, V., Soatto, S.: Causal video object segmentation from persistence of occlusions. In: Proceedings of the IEEE Conference on Computer Vision and Pattern Recognition. (2015) 4268-4276

22. Fragkiadaki, K., Zhang, G., Shi, J.: Video segmentation by tracing discontinuities in a trajectory embedding. In: Computer Vision and Pattern Recognition (CVPR), 2012 IEEE Conference on, IEEE (2012) 1846-1853

23. Sawhney, H.S., Guo, Y., Asmuth, J., Kumar, R.: Independent motion detection in 3d scenes. In: Computer Vision, 1999. The Proceedings of the Seventh IEEE International Conference on. Volume 1., IEEE (1999) 612-619

24. Dey, S., Reilly, V., Saleemi, I., Shah, M.: Detection of independently moving objects in non-planar scenes via multi-frame monocular epipolar constraint. In: Computer Vision-ECCV 2012. Springer (2012) 860-873

25. Namdev, R.K., Kundu, A., Krishna, K.M., Jawahar, C.V.: Motion segmentation of multiple objects from a freely moving monocular camera. In: International Conference on Robotics and Automation. (2012)

26. Elhamifar, E., Vidal, R.: Sparse subspace clustering. In: Computer Vision and Pattern Recognition, 2009. CVPR 2009. IEEE Conference on, IEEE (2009) 27902797

27. Horn, B.K.: Projective geometry considered harmful (1999)

28. Jepson, A.D., Heeger, D.J.: Linear subspace methods for recovering translational direction. In: Proceedings of the 1991 York conference on Spatial vision in humans and robots, New York, NY, USA, Cambridge University Press (1993) 39-62

29. Prazdny, K.: On the information in optical flows. Computer Vision, Graphics, and Image Processing 22(2) (1983) 239-259

30. Tomasi, C., Shi, J.: Direction of heading from image deformations. In: CVPR. (1993)

31. Prazdny, K.: Egomotion and relative depth map from optical flow. Biological Cybernetics 36(2) (1980) 87-102

32. Tian, T., Tomasi, C., Heeger, D.: Comparison of approaches to egomotion computation. In: CVPR. (1996)

33. Yamaguchi, K., McAllester, D., Urtasun, R.: Robust monocular epipolar flow estimation. In: CVPR. (2013)

34. Hartley, R., et al.: In defense of the eight-point algorithm. Pattern Analysis and Machine Intelligence, IEEE Transactions on 19(6) (1997) 580-593

35. Horn, B.: Robot vision. MIT Press (1986)

36. Bruss, A.R., Horn, B.K.: Passive navigation. Computer Vision, Graphics, and Image Processing 21(1) (1983) 3-20

37. Sun, D., Roth, S., Black, M.J.: Secrets of optical flow estimation and their principles. In: Computer Vision and Pattern Recognition (CVPR), 2010 IEEE Conference on, IEEE (2010) 2432-2439

38. Narayana, M., Hanson, A., Learned-Miller, E.G.: Background subtraction: separating the modeling and the inference. Machine vision and applications 25(5) (2014) 1163-1174

39. Fischler, M.A., Bolles, R.C.: Random sample consensus: a paradigm for model fitting with applications to image analysis and automated cartography. Communications of the ACM 24(6) (1981) 381-395 
40. Achanta, R., Shaji, A., Smith, K., Lucchi, A., Fua, P., Susstrunk, S.: Slic superpixels compared to state-of-the-art superpixel methods. Pattern Analysis and Machine Intelligence, IEEE Transactions on 34(11) (2012) 2274-2282

41. Otsu, N.: A threshold selection method from gray-level histograms. IEEE Transactions on Systems, Man, and Cybernetics 9 (1979) 62-66

42. Wang, J.Y., Adelson, E.H.: Representing moving images with layers. Image Processing, IEEE Transactions on 3(5) (1994) 625-638

43. Powers, D.M.: Evaluation: from precision, recall and f-measure to roc, informedness, markedness and correlation. Technical Report Technical Report SIE-07-001, Flinders University, Adelaide (2007)

44. : Most amazing camouflage (part 1 of 3 ). http://www.youtube. com/watch?v= Wc5wMX61FZ8 (2014) [Online; accessed 1-March-2015].

45. : Most amazing camouflage (part 2 of 3). https://www.youtube.com/watch?v= yoG1P4new04 (2014) [Online; accessed 1-March-2015].

46. : Most amazing camouflage (part 3 of 3 ). https://www.youtube.com/watch?v= GnEkBJnS_FU (2014) [Online; accessed 1-March-2015].

47. : Phasmid - incredible insect camouflage. https://www.youtube. com/watch?v= adufPBDNCKo (2014) [Online; accessed 1-March-2015]. 


\section{Supplementary Material}

Our supplementary material contains:

- a review of the BMS-26 data set and a detailed overview about excluded video sequences,

- additional details about the Camouflaged Animals Data Set,

- related work about camera motion estimation and

- a description of our modified Bruss and Horn error [36], a fundamental part of our motion segmentation initialization.

\section{Comparability of the motion segmentation data sets}

A lot of different databases have been created to provide a common benchmark for motion segmentation. Often a clear definition of what people intend to segment when they provide a ground truth is missing. This results in many inconsistent segmentations, which makes it hard to compare against other motion segmentation methods. In our paper we give a clear definition of motion segmentation

(I) Every pixel is given one of two labels: static background or moving objects.

(II) If only part of an object is moving, the entire object should be segmented.

(III) All freely moving objects should be segmented, but nothing else.

(IV) Stationary objects are not segmented, even when they moved before or will move in future. We consider segmentation of previously moving objects to be tracking.

In the following subsection we give detailed information about all videos that do not correspond to our understanding of motion segmentation.

\section{Berkeley Motion Segmentation database (BMS-26)}

To satisfy the first criterion of motion segmentation we converted the given ground truth into a binary segmentation, removing the provided motion labels. If all four criteria are satisfied, we used the video for comparison. The effect of the mislabeled ground truth varies a lot. The difference between a correct ground truth of marple 2 and the provided ground truth for example is enormous, whereas the difference of a correct ground truth of tennis would be almost not noticeable. Trying to be as objective as possible we excluded all videos where one of our four criteria of motion definition is violated, indepently of the size of the mislabeled region. The following table shows the sequences we excluded for evaluation. 


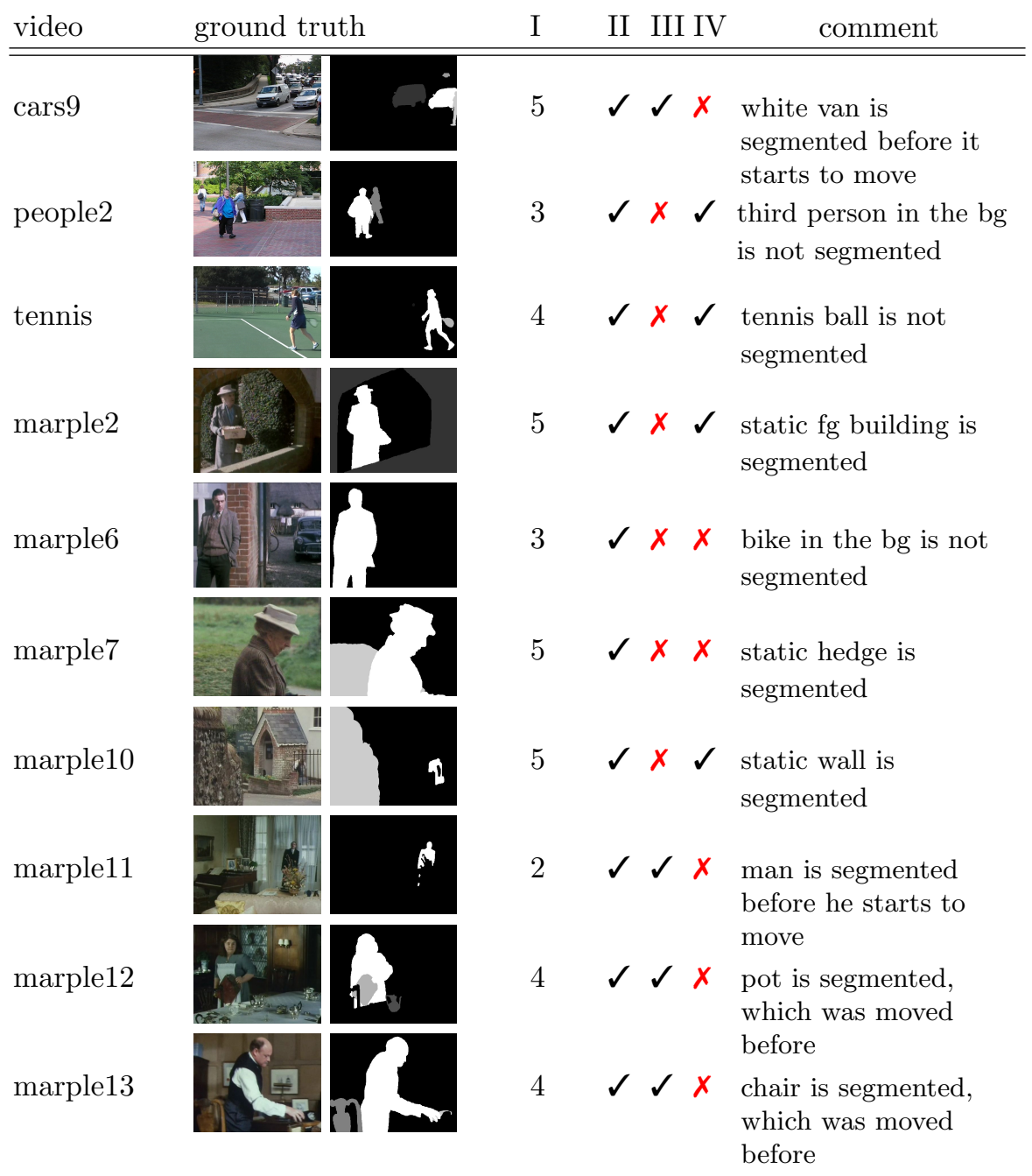

Table 3: Ground truth of BMS-26 video sequences we excluded for evaluation due to mislabeled regions in ground truth.

\section{Camouflaged Animals Data Set}

Our new data set includes nine short video sequences extracted from YouTube videos and an accompanying ground truth. Table 1 shows the link to the original YouTube video, the exact time where our chosen video sequence starts within the YouTube video, and the number of frames the sequence contains. For our motion segmentation algorithm, we converted the video sequence to an image sequence in the png format using the VideoReader function of Matlab. Each sequence contains hand-labeled ground truth of moving objects in every fifth frame. 


\begin{tabular}{|c|c|c|c|c|}
\hline Video & Link & Start & Frames \\
\cline { 1 - 2 } chameleon & $\overline{44}$ & $02: 28.20$ & 218 \\
frog & $\overline{45}$ & $00: 05.38$ & 31 \\
glow-worm beetle & $\overline{\overline{46}}$ & $06: 02.13$ & 104 \\
snail & $\overline{46}$ & $06: 07.02$ & 84 \\
scorpion1 & $\overline{46}$ & $02: 09.00$ & 105 \\
scorpion2 & $\overline{46}$ & $02: 25.04$ & 61 \\
scorpion3 & $\overline{46}$ & $02: 27.48$ & 76 \\
scorpion4 & $\overline{46}$ & $00: 06.11$ & 80 \\
stickinsect & $\overline{47}$ & $00: 05.68$ & 80 \\
\hline
\end{tabular}

\section{Camera motion estimation in the context of motion segmentation}

In camera motion estimation, also known as egomotion estimation, the relationship between the observed optical flow and the camera's translation and rotation are exploited in different ways to estimate the six motion parameters. Jepson and Heeger 28 construct a set of constraints for optical flow observations in a manner that effectively negates the rotation component. Prazdny 29] shows that the difference between any two flow vectors yields a constraint that does not depend on rotation. Tomasi and Shi 30 estimate translation by using image deformation rather than movement of points within an image, to minimize the effects of rotation. In the above methods, translation is first estimated using the rotation invariant constraints, followed by rotation estimation. Prazdny 31 estimates the rotation first by using constraints that are independent of camera translation and the depth of the points in the scene. These and other methods for egomotion estimation are described by Tian et al. 32. Recently, many authors (e.g. 33]) have used projective geometry methods such as the 8-point algorithm 34 in combination with RANSAC [39] for robustness.

We are not directly interested in computing the egomotion, but rather in transforming the observed optical flow vectors such that the flow vectors are a function of only translation, and not rotation. This is similar in spirit to Yamaguchi et al. [33, who estimate the rotation at each pixel such that when the rotational flow vectors are subtracted from the observed flow vectors, a pure translational flow field is obtained. But they do this under the assumption of a static scene. We demonstrate successful motion modeling in the presence of multiple large moving objects in Section 3 of our paper. Based on a least squares minimization to find the camera translation [36] we slightly modify the error function to apply the camera motion estimation problem to the motion segmentation problem. Furthermore we add a RANSAC procedure as initialization for robustness and outlier reduction.

\section{Modified Bruss and Horn Error}

As described in the main text, we introduced a modification to the error function of the Bruss and Horn algorithm that we call the modified Bruss and Horn $(\mathrm{MBH})$ error. We first give some basic background on perspective projection, 
describe the Bruss and Horn error function and a particular issue that makes it problematic in the context of motion segmentation, and then describe our modification to the algorithm.

Basics of perspective projection. Given a particular set of translation parameters $(U, V, W)$ (and assuming no camera rotation), the direction of optical flow of the background can be predicted for each point $(x, y)$ in the image via the perspective projection equations. Let a point $P$ have $3-\mathrm{D}$ coordinates $(X, Y, Z)$. Then the image coordinates $(x, y)$ for this point are:

$$
x=\frac{X f}{Z} \quad \text { and } \quad y=\frac{Y f}{Z},
$$

where $f$ is the camera's focal length. A tranlational camera motion $(U, V, W)$ yields in a pixel displacment $\boldsymbol{v}$ in the image.

$$
v_{x}=\frac{W \cdot x-U \cdot f}{Z} \quad \text { and } \quad v_{y}=\frac{W \cdot y-V \cdot f}{Z} .
$$

The direction of the motion, given by

$$
\arctan (W \cdot y-V \cdot f, W \cdot x-U \cdot f)
$$

is then a function of the original image position $(x, y)$, the direction of motion $(U, V, W)$ and the focallength $f$, and has no dependence on the depth $Z$ of the point.

Fig. 9: Bruss and Horn error. Let $\boldsymbol{p}$ be a vector in the direction of preferred motion with respect to a motion hypothesis $(U, V, W)$. The Bruss and Horn error assigned to a translational flow vector $\boldsymbol{v}_{t}$ is then the distance of its projection onto $\boldsymbol{p}$. However, this same error would be assigned to a vector $-\boldsymbol{v}_{t}$ pointing in the opposite direction, which should have much lower compatibility with the motion hy-

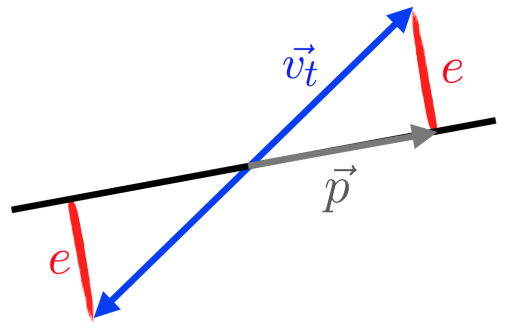
pothesis.

The Bruss and Horn Error Function. The point of the Bruss and Horn algorithm (translation-only case) is to find the motion direction parameters $(U, V, W)$ that are as compatible as possible with the observed optical flow vectors. Let $\boldsymbol{p}$ be a vector in the direction of the flow expected from a motion $(U, V, W)$ (see Figure 9). Then the Bruss and Horn error for the observed flow vector $\boldsymbol{v}_{t}$ is the distance of the projection of $\boldsymbol{v}_{t}$ onto $\boldsymbol{p}$, shown by the red segment $e$ on the right side of the figure.

The problem with this error function is that this distance is small not only for vectors which are close to the preferred direction, but also for vectors that are in a direction opposite the preferred direction. That is, observed optical flow vectors that point in exactly the wrong direction with respect to a motion $(U, V, W)$ get 
a small error in the Bruss and Horn algorithm. In particular, the error assigned to a vector $\boldsymbol{v}_{t}$ is the same as the error assigned to a vector $-\boldsymbol{v}_{t}$ in the opposite direction (See Figure 9).

Because the Bruss and Horn algorithm is intended for motion estimation in scenarios where there is only a single moving object (the background), such motions in the opposite direction to the preferred motion are not common, and thus, this "problem" we've identified has little impact. However, in the motion segmentation setting, where flows of objects may be in opposite directions, this can make the flow of a separately moving object (like a car), look as though it is compatible with the background. We address this problem by introducing a modified version of the error.

The modified Bruss and Horn error. As stated above, the Bruss and Horn error is the distance of the projection of an optical flow vector onto the vector $\boldsymbol{p}$ representing the preferred direction of flow according to a motion model $(U, V, W)$. This can be written simply as

$$
e_{B H}\left(\boldsymbol{v}_{t}, \boldsymbol{p}\right)=\left\|\boldsymbol{v}_{t}\right\| \cdot \mid \sin \left(\measuredangle\left(\boldsymbol{v}_{t}, \boldsymbol{p}\right) \mid .\right.
$$

This error function has the appropriate behavior when the observed optical flow is within 90 degrees of the expected flow direction, i.e., when $\boldsymbol{v}_{t} \cdot \boldsymbol{p} \geq 0$. However, when the observed flow points away from the preferred direction, we assign an error equal to the magnitude of the entire vector, rather than its projection, since no component of this vector represents a "valid direction" with respect to $(U, V, W)$. This results in the modified Bruss and Horn error (see Figure 10:

Fig. 10: Modified Bruss and Horn error. When an observed translation vector $\boldsymbol{v}_{t}$ is within 90 degrees of the preferred direction, its error is computed in the same manner as the traditional Bruss and Horn error (right side of figure). However, when the observed vector is more than 90 degrees from the preferred direction, its error is computed as its full magnitude, rather than the distance of projection (left side of figure). This new error function keeps objects moving in opposite directions from being confused with each other.

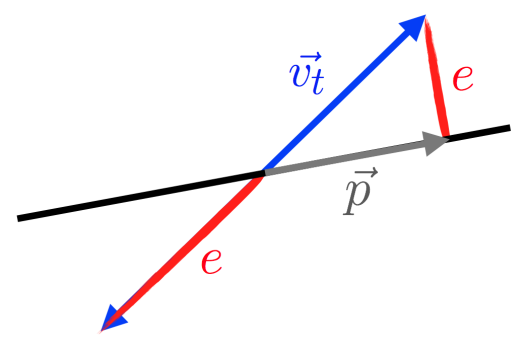

$$
e_{M B H}= \begin{cases}\left\|\boldsymbol{v}_{t}\right\|, & \text { if } \boldsymbol{v}_{t} \cdot \boldsymbol{p}<0 \\ \left\|\boldsymbol{v}_{t}\right\| \cdot \mid \sin \left(\measuredangle\left(\boldsymbol{v}_{t}, \boldsymbol{p}\right) \mid,\right. & \text { otherwise. }\end{cases}
$$

This error has the desired behavior of penalizing flows in the opposite direction to the expected flow. 